\title{
Luminescence quenching by photoionization and electron transport in a $\mathrm{LaAlO}_{3}: \mathrm{Ce}^{3+}$ crystal
}

\author{
E. van der Kolk, ${ }^{\text {a) }}$ J. T. M. de Haas, A. J. J. Bos, C. W. E. van Eijk, and P. Dorenbos \\ Faculty of Applied Sciences, Delft University of Technology, Mekelweg 15, $2629 \mathrm{JB}$ Delft, \\ The Netherlands
}

(Received 27 June 2006; accepted 31 January 2007; published online 18 April 2007)

\begin{abstract}
A combined photoconductivity, absorption, and thermoluminescence study was performed to understand the absence of luminescence from $\mathrm{Ce}^{3+}$ in $\mathrm{LaAlO}_{3}: \mathrm{Ce}^{3+}$. It is demonstrated that the absence of luminescence is the result of $\mathrm{Ce}^{3+}$ ionization from the $5 d$ excited states, which are all located in the conduction band. $\mathrm{Ce}^{3+}$ ionization is accompanied by the formation of several broad absorption and photoconductivity bands, which are assigned to electron traps. A time and temperature dependent optical investigation of these traps reveals the conditions under which electrons are transferred from $\mathrm{Ce}^{3+}$ to traps and vice versa, from traps back to $\mathrm{Ce}^{4+}$. The observed difference in energy needed to thermally or optically release electrons from traps is qualitatively explained in terms of the location of the ground and excited states of the electron traps with respect to the conduction band. (C) 2007 American Institute of Physics. [DOI: 10.1063/1.2715748]
\end{abstract}

\section{INTRODUCTION}

It is well known that some $\mathrm{Ce}^{3+}$ doped wide band-gap insulators such as $\mathrm{Y}_{2} \mathrm{O}_{3}, \mathrm{Lu}_{2} \mathrm{O}_{3}$, and $\mathrm{La}_{2} \mathrm{O}_{3}$ do not show any $\mathrm{Ce}^{3+}$ luminescence, not even at liquid helium temperature. In such wide band-gap hosts, $\mathrm{Ce}^{3+}$ has a $[\mathrm{Xe}] 4 f^{1}$ electron configuration with a $[\mathrm{Xe}] 4 f^{0} 5 d^{1}$ excited state configuration at typically $2-3 \mathrm{eV}$ higher energy. Because of this large energy gap, direct nonradiative inter-configurational relaxation from the excited $5 d$ state to the $4 f$ ground state is inefficient, so that $\mathrm{Ce}^{3+}$ usually shows luminescence with high quantum efficiency. Absence of $5 d \rightarrow 4 f$ luminescence of $\mathrm{Ce}^{3+}$ therefore strongly suggests an alternative nonradiative luminescence quenching route. Photoconductivity studies on $\mathrm{Lu}_{2} \mathrm{O}_{3}: \mathrm{Ce}^{3+}$ by Raukas et al. ${ }^{1}$, Yen et al., ${ }^{2,3}$ and Happek et $\mathrm{al}^{4}{ }^{4}$ demonstrated that luminescence quenching proceeds via $\mathrm{Ce}^{3+}$ ionization and subsequent nonradiative recombination between $\mathrm{Ce}^{4+}$ and the electron. The preferred nonradiative ionization route was explained by the unfavorable location of the lowest energy $5 d$ state in the conduction band (CB) of $\mathrm{Lu}_{2} \mathrm{O}_{3}$. $\mathrm{LaAlO}_{3}: \mathrm{Ce}^{3+}$ is another material in which $\mathrm{Ce}^{3+}$ luminescence has never been observed. ${ }^{5}$ Contrary to a recent report by Zeng et al., ${ }^{6}$ we were not able to detect $\mathrm{Ce}^{3+}$ luminescence in $\mathrm{LaAlO}_{3}$. In the first part of this work, we present a combined photoconductivity, absorption, and thermoluminescence study and show that the absence of luminescence is caused, as in $\mathrm{Lu}_{2} \mathrm{O}_{3}$ by $\mathrm{Ce}^{3+}$ ionization. $\mathrm{Ce}^{3+}$ ionization was not only evidenced by the detection of a photocurrent but also by the formation of color centers related to electron traps. Electron traps could be observed directly in photocurrent and absorption spectra as well as indirectly by thermo luminescence glow curve analysis. For this reason, the second part of this work is concerned with the formation and successive optically or thermally stimulated dissociation of electron traps.

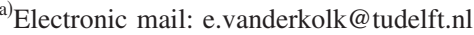

\section{EXPERIMENTAL METHODS}

The experimental facility to record photocurrent excitation spectra was described before. ${ }^{7}$ Absorption spectra between 200 and $800 \mathrm{~nm}$ were obtained with an HP 8452A diode array spectrophotometer. Temperature dependence of the absorption spectra between 80 and $800 \mathrm{~K}$ was achieved by insertion of a Janis VPF-700 liquid nitrogen cryostat. The absorption spectra could be obtained before, during, or after illumination of the sample with light from a $150 \mathrm{~W}$ xenon lamp. Light from the xenon lamp was focused, using two lenses, onto the sample via a small mirror mounted inside the sample chamber of the cryostat. The illumination wavelength selection between 250 and $650 \mathrm{~nm}$ was achieved by using $10 \mathrm{~nm}$ full width of half maximum (FWHM) interference filters with a transmission of about $10 \%$. Thermoluminescence (TL) glow curves were recorded as a function of stimulation wavelength with a TL/optically stimulated luminescence (OSL) system TL-DA-15 from RIS $\varnothing$ national laboratory. ${ }^{8}$ More details about this type of measurement will be given Sec. III. One clear $\mathrm{Ce}^{3+}$ doped $\mathrm{LaAlO}_{3}$ crystal was available for study. The $\mathrm{Ce}^{3+}$ concentration was estimated to be about $1 \%-2 \%$ based on our absorption measurements and typical $\mathrm{Ce}^{3+} 4 f \rightarrow 5 d$ absorption coefficients of about $100 \mathrm{~cm}^{-1}$. $\mathrm{LaAlO}_{3}$ adopts the rhombohedral perovskite structure with space group $R \overline{3} c{ }^{9}$

\section{EXPERIMENTAL RESULTS AND DISCUSSION}

Figure 1 compares the photocurrent excitation spectrum, recorded from low to high energy (solid line) with the absorption spectrum (dotted line) of a $\mathrm{LaAlO}_{3}: \mathrm{Ce}^{3+}$ crystal. The spectra are almost identical with two bands centered around 315 and $245 \mathrm{~nm}$ corresponding to the doublet and triplet $5 d$ states of $\mathrm{Ce}^{3+}$, respectively. ${ }^{5}$ The splitting of the doublet state as observed in the photocurrent excitation spectrum is believed to result from a saturation effect of the photocurrent detection efficiency at high absorption strength. 


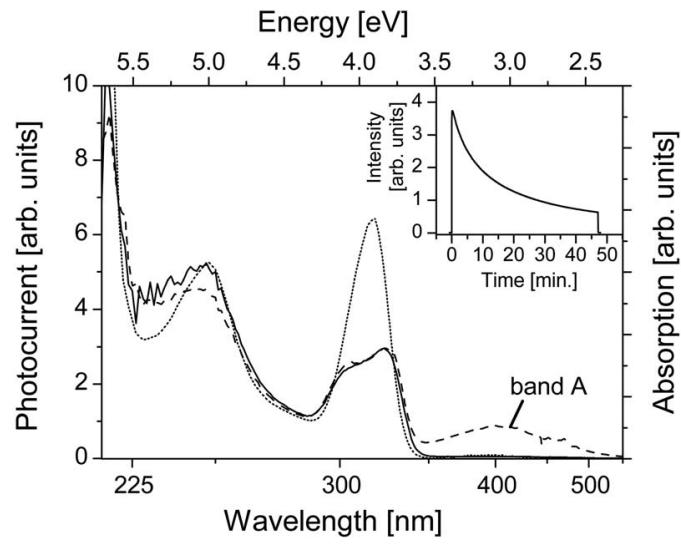

FIG. 1. Photocurrent excitation spectrum (solid), absorption spectrum (dotted), and a second photocurrent excitation spectrum (dashed) of $\mathrm{LaAlO}_{3}: \mathrm{Ce}^{3+}$ recorded at room temperature. The inset shows the time dependence of the photocurrent during continuous $420 \mathrm{~nm}$ excitation.

The saturation effect was systematically studied by the author in $\mathrm{a} \mathrm{Lu}_{2} \mathrm{SiO}_{5}: \mathrm{Ce}^{3+}$ crystal as a function of crystal thickness. ${ }^{10}$ In this study, it appeared that saturation is strong in optically dense crystals, while it is absent in optically thin crystals. The intensities of the photocurrent excitation bands did not change as a function of temperature within the investigated temperature range between 80 and $450 \mathrm{~K}$. The onset at $225 \mathrm{~nm}$ that can be seen in the photocurrent as well as in the absorption spectrum is assigned to the fundamental absorption edge of $\mathrm{LaAlO}_{3}{ }^{5}$

Previous temperature dependent photoconductivity experiments on $\mathrm{Lu}_{2} \mathrm{SiO}_{5}: \mathrm{Ce}^{3+}, 111 M_{2} \mathrm{SiO}_{5}: \mathrm{Ce}^{3+}, \mathrm{Pr}^{3+}$ (with $M=\mathrm{Y}$ or $\mathrm{Lu}),{ }^{12}$ and $\mathrm{GdAlO}_{3}: \mathrm{Ce}^{3+}$ Ref. 7 have demonstrated that when the lowest energy $5 d$ state of $\mathrm{Ce}^{3+}$ is located below but close to the conduction band minimum (typically $0.5-0.1 \mathrm{eV}$ ), the photocurrent intensity upon $5 d$ excitation becomes strongly temperature dependent due to a thermally stimulated ionization process. Other studies ${ }^{13-15}$ have shown that when the lowest energy $5 d$ states are far below the conduction band minimum (typically $>1 \mathrm{eV}$ ), $5 d$ states do not show up in the photocurrent excitation spectrum. The fact that the absorption and photocurrent excitation spectra of $\mathrm{LaAlO}_{3}: \mathrm{Ce}^{3+}$ are identical and that the photocurrent spectrum is temperature independent suggests that the lowest energy $5 d$ state $\mathrm{Ce}^{3+}$ is located inside the $\mathrm{CB}$ of $\mathrm{LaAlO}_{3}$. This interpretation explains the absence of $\mathrm{Ce}^{3+}$ luminescence in this material at a temperature as low as $10 \mathrm{~K}$ and is in line with the conclusions on $\mathrm{Lu}_{2} \mathrm{O}_{3}: \mathrm{Ce}^{3+}$ Refs. 1-4 discussed in Sec. I.

Figure 1 contains a second photocurrent excitation spectrum (dashed line) that was recorded immediately after the first photocurrent spectrum (solid curve), also from low to high energy. In this spectrum a different broad band centered around $400 \mathrm{~nm}$ can be observed which was absent in the first scan. Apparently, UV exposure during the first scan has created color centers that participated in the photocurrent process during the second scan. The shape of this band, which will be referred to as band $A$ for convenience, is affected strongly by the nonpersistent nature of the photocurrent when this band is excited. The inset of Fig. 1 explicitly shows the nonpersistent nature of the photocurrent when

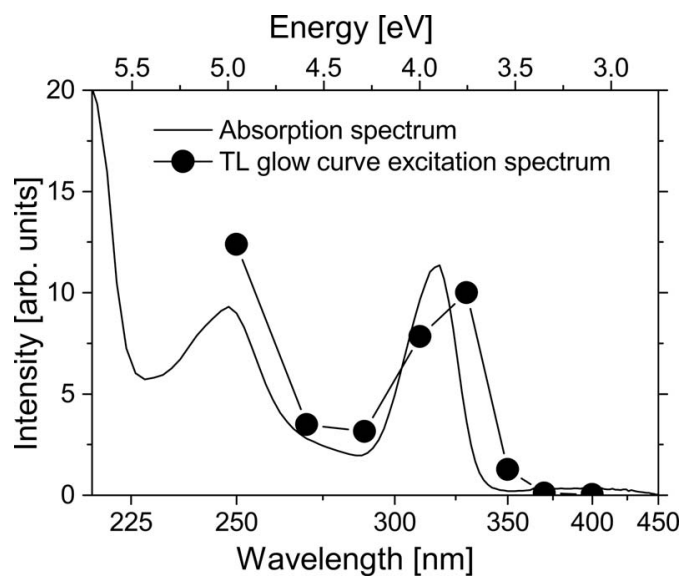

FIG. 2. Room temperature absorption spectrum (solid) and thermoluminescence glow curve excitation spectrum (dots) of $\mathrm{LaAlO}_{3}: \mathrm{Ce}^{3+}$.

band $A$ is excited at $420 \mathrm{~nm}$. The photocurrent intensity as a function of time is not constant but shows a no-exponential decay. As a result of this nonpersistent behavior, the previously assigned $400 \mathrm{~nm}$ maximum of band $A$ is rather arbitrary and changes when experimental parameters such as excitation intensity or scan speed are modified. It is plausible that band $A$ corresponds to excitation of electrons from intrinsic traps of the host lattice that are filled during $\mathrm{Ce}^{3+}$ ionization.

To confirm that band $A$ really is created by $\mathrm{Ce}^{3+}$ ionization and subsequent electron trapping, TL glow curves were recorded as a function of illumination wavelength to obtain something that may be called a TL glow curve excitation spectrum. For a more detailed explanation of this type of measurement, see Refs. 16 and 17 wherein it is called photostimulated trap filling or thermally stimulated luminescence excitation spectroscopy (TSLES). In our case, the TL glow curve excitation spectrum was obtained by illuminating the sample at room temperature for a duration of $10 \mathrm{~s}$ with a $150 \mathrm{~W}$ xenon lamp through a $10 \mathrm{~nm}$ FWHM interference filter. Next, a TL glow curve between room temperature and $450 \mathrm{~K}$ was recorded. In Fig. 2 the temperature integrated TL intensities obtained using band filters transmitting at 250, $270,290,310,330,350,370,400$, and $450 \mathrm{~nm}$ are plotted (dotted curve) and compared with the absorption spectrum (solid curve). The resemblance of the absorption spectrum and the TL creation spectrum demonstrates quite convincingly that traps that are emptied during the TL glow curve were filled through $\mathrm{Ce}^{3+} 5 d$ excitation. This indirect detection of electron transport from $\mathrm{Ce}^{3+}$ to traps is in accordance with the observed photocurrent when $\mathrm{Ce}^{3+}$ is excited (Fig. 1). Vice versa, the observed nonpersistent photocurrent that is detected when band $A$ is excited shows that trapped electrons can be excited optically into the $\mathrm{CB}$ and transferred back to $\mathrm{Ce}^{4+}$.

The TL experiments show that the electron traps and the corresponding $\mathrm{Ce}^{4+}$ ions are thermally stable at least for several minutes between light stimulation and the TL measurement. In order to investigate the thermal stability of band $A$ in more detail, time and temperature dependent absorption spectra were recorded. The experimental facility to record absorption spectra was modified, as described in Sec. II, in 


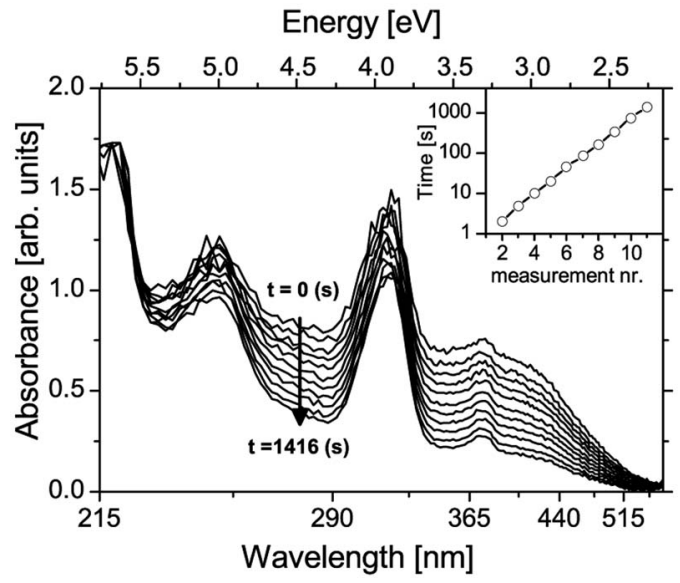

FIG. 3. Time dependence of the absorption spectrum of $\mathrm{LaAlO}_{3}: \mathrm{Ce}^{3+}$ at room temperature after $310 \mathrm{~nm}$ illumination is switched off.

such a way that absorption could be measured before, during, and after optical illumination of the crystal. Figure 3 shows time dependent absorption spectra recorded at room temperature. Before the spectra in Fig. 3 were recorded, the sample was illuminated with $310 \mathrm{~nm}$ light until the intensity of band $A$ saturated at a maximum value. After the $310 \mathrm{~nm}$ illumination was switched of, absorption spectra were recorded at different moments with increasing time intervals at $0,2,5$, 10, 20, 45, 86, 166, 345, 750, and 1416 s (see inset). Each absorption spectrum was recorded within a $0.25 \mathrm{~s}$ integration time of the diode array. Figure 3 shows that band $A$ is not stable at room temperature, which is in accordance with the absence of band $A$ in the first photocurrent spectrum (Fig. 1, solid curve).

Figure 4 represents the time dependence of the intensity of band $A$ at $420 \mathrm{~nm}$ at different temperatures. Curve 1 was recorded at $295 \mathrm{~K}$ and represents the intensity of band $A$ at $420 \mathrm{~nm}$ as a function of time. These data were taken from Fig. 3. Curves 2, 3, and 4 represent the same measurement at 400,500 , and $600 \mathrm{~K}$. Each measurement was started the moment that the $310 \mathrm{~nm}$ light stimulation was switched off. It is observed that the bleaching of band $A$ is much faster at higher temperature than at lower temperature, and is therefore clearly thermally activated. The time dependence does

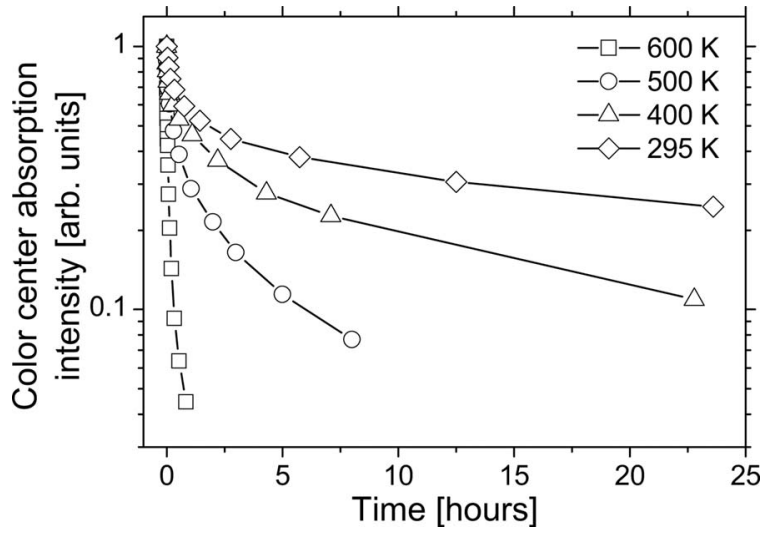

FIG. 4. Temperature dependence of fading of the color center absorption intensity at $420 \mathrm{~nm}$ of $\mathrm{LaAlO}_{3}: \mathrm{Ce}^{3+}$ after $310 \mathrm{~nm}$ stimulation was switched off.

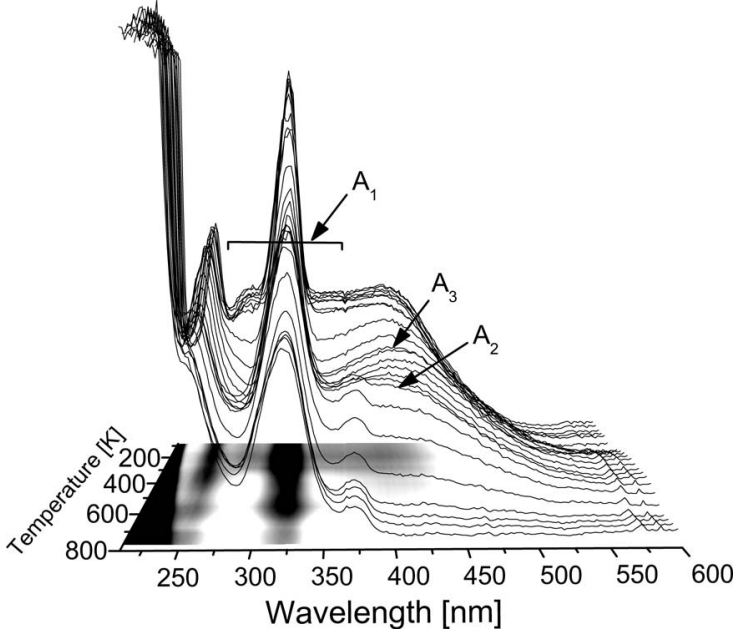

FIG. 5. Waterfall plot with surface projection of the absorption spectrum of $\mathrm{LaAlO}_{3}: \mathrm{Ce}^{3+}$ between 80 and $770 \mathrm{~K}$, recorded during continuous $310 \mathrm{~nm}$ illumination.

not follow a simple exponential behavior at the temperatures measured. The nonexponential detrapping behavior may be explained by retrapping of electrons or the presence of more than one type of trap with different trap depths. A rough estimate of the deepest trap depth energy $E_{\text {trap }}$ can be obtained by fitting curves 1-4 (see Fig. 4) between the last two data points with a single exponential function $\exp [-t / \tau]$. $E_{\text {trap }}$ can be found by using the well known Arrhenius formula for a thermally stimulated process. From such a fit, a trap depth of about $0.8 \mathrm{eV}$ follows.

Figure 5 presents a waterfall plot with surface projection of the temperature dependence of the absorption spectrum recorded between 80 and $770 \mathrm{~K}$ and 200 and $650 \mathrm{~nm}$. In order to record the absorption spectrum with the highest possible color center absorption intensity (band $A$ ), all spectra in Fig. 5 were collected during continuous stimulation with $310 \mathrm{~nm}$ light. The integration time of the diode array was set at $1 \mathrm{~s}$. At the highest measured temperature of $770 \mathrm{~K}, \mathrm{Ce}^{3+}$ $4 f \rightarrow 5 d$ absorption is observed, but there are no color center related absorption bands. At low temperature $(80 \mathrm{~K})$, on the other hand, the intensity of band $A$ is high. Following the spectra from low to high temperature, it can be noticed that band $A$ actually consists of at least three bands, each with its own characteristic quenching temperature and center wavelength. From $290 \mathrm{~K}$ onwards, a band around $310 \mathrm{~nm}$, below $\mathrm{Ce}^{3+} 4 f \rightarrow 5 d$ absorption (see arrow in Fig. 5), starts to lose intensity. This band called $A_{1}$ is fully bleached at $380 \mathrm{~K}$. A second band $A_{2}$, around $425 \mathrm{~nm}$, starts to bleach at slightly higher temperature $(\sim 410 \mathrm{~K})$ and has lost its intensity around $500 \mathrm{~K}$. A third band $A_{3}$, centered around $400 \mathrm{~nm}$, starts to bleach at $560 \mathrm{~K}$. Above about $700 \mathrm{~K}$, all broad band features are quenched despite continuous $\mathrm{Ce}^{3+} 5 d$ excitation at $310 \mathrm{~nm}$. We have no explanation for the small band centered around $370 \mathrm{~nm}$ that appeared above $500 \mathrm{~K}$.

It must be noted that the intensity of the absorption features related to the electron traps depend on the crystal growth conditions. Zeng et al. ${ }^{6}$ showed that $\mathrm{Ce}^{3+}$ doped and undoped $\mathrm{LaAlO}_{3}$ crystals have broad absorption bands in the region of band $A$ that can be removed partly by annealing in a $\mathrm{H}_{2}$ atmosphere. 


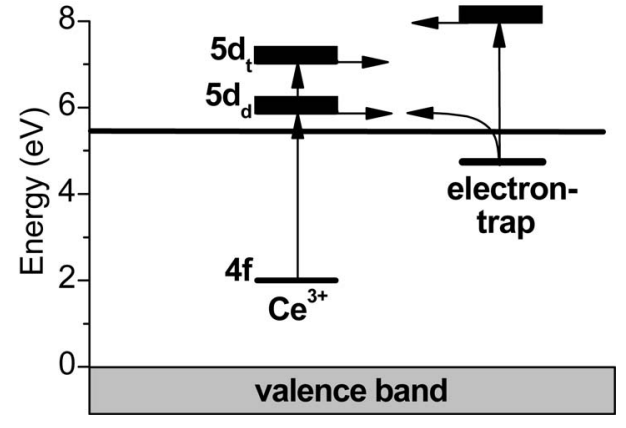

FIG. 6. Schematic energy level scheme of $\mathrm{LaAlO}_{3}: \mathrm{Ce}^{3+}$. The ground and excited states of $\mathrm{Ce}^{3+}$ and an electron trap are plotted relative to the $\mathrm{CB}$ and VB.

It can be concluded that the energy needed to empty traps optically via bands $A_{1}(4.0 \mathrm{eV}), A_{2}(2.9 \mathrm{eV})$, and $A_{3}$ $(3.3 \mathrm{eV})$ into the $\mathrm{CB}$ is significantly larger than the thermal energy that is needed to release an electron from its trap $(0.8 \mathrm{eV})$. This can be explained as follows. The thermally activated process proceeds from the electron trap ground state via the conduction band minimum. The optical recombination, on the other hand, takes place from the ground state via the excited states of the electron taps that are located in the conduction band. The optically stimulated electron-Ce $\mathrm{e}^{4+}$ recombination process therefore requires more energy than the thermally activated process.

The surface projection of Fig. 5 was added because it clearly reveals the temperature dependence of the absorption features of $\mathrm{LaAlO}_{3}: \mathrm{Ce}^{3+}$. Firstly, the lowering in energy of the fundamental absorption of $\mathrm{LaAlO}_{3}$ with increasing temperature of $d E / d T=-0.8 \mathrm{meV} / \mathrm{K}$ is deduced. Secondly, it is seen that the absorption energy of the triplet and doublet states of the $5 d$ configuration of $\mathrm{Ce}^{3+}$ is less dependent on temperature and shifts to lower energy from 5.04 and $3.95 \mathrm{eV}$ at $80 \mathrm{~K}$ to about 4.90 and $3.87 \mathrm{eV}$ at $770 \mathrm{~K}$. The width of the doublet absorption band increases from $0.24 \mathrm{eV}$ FWHM at $80 \mathrm{~K}$ to $0.60 \mathrm{eV}$ FWHM at $770 \mathrm{~K}$. These data suggest that the shirinking of the band gap toward higher temperature may have a significant effect on the relative location of the $5 d$ states of $\mathrm{Ce}^{3+}$ and the bottom of the conduction band.

\section{SUMMARY}

The experimental results discussed in the previous section are summarized in a schematic energy level diagram of $\mathrm{LaAlO}_{3}: \mathrm{Ce}^{3+}$ (see Fig. 6). The separation of $5.5 \mathrm{eV}$ between the $\mathrm{CB}$ and valence band (VB) was deduced from the absorption and photocurrent spectra (Fig. 1). The $5 d$ states of $\mathrm{Ce}^{3+}$ are placed in the $\mathrm{CB}$ on the basis of the photocurrent and TL creation spectra. The doublet state is placed arbitrarily a little bit above the CB bottom. The triplet state and the ground state are placed using the absorption spectrum of Fig. 1. Based on the thermal bleaching behavior of the absorption bands related to the electron traps (Fig. 4), we have placed the ground state of an electron trap at $0.8 \mathrm{eV}$ below the $\mathrm{CB}$ bottom. One of the excited states of the electron traps is placed in the $\mathrm{CB}$, on the basis of the absorption data of Fig. 5 , at $3.3 \mathrm{eV}$ above the electron trap ground state. Straight arrows represent optically stimulated ionization processes, while curved arrows indicate thermally stimulated processes. This energy level structure results in the unusual behavior of $\mathrm{LaAlO}_{3}: \mathrm{Ce}^{3+}$, in which $5 d$ excitation does not result in $\mathrm{Ce}^{3+}$ luminescence but in $\mathrm{Ce}^{3+}$ ionization and the filling of electron traps. Depending on the energy of optical stimulation (below or above $\sim 3.4 \mathrm{eV}$ ), electrons can be transferred back and forth between $\mathrm{Ce}^{3+}$ and the electron traps.

\section{ACKNOWLEDGMENT}

This work was supported by the Dutch Technology Foundation (STW).

${ }^{1}$ M. Raukas, S. A. Basun, W. van Schaik, W. M. Yen, and U. Happek, Appl. Phys. Lett. 69, 3300 (1996).

${ }^{2}$ W. M. Yen, M. Raukas, S. A. Basun, W. van Schaik, and U. Happek, J. Lumin. 69, 287 (1996).

${ }^{3}$ W. M. Yen, Mater. Res. Soc. Symp. Proc. 560, 183 (1999).

${ }^{4}$ U. Happek, S. A. Basun, J. Choi, J. K. Krebs, and M. Raukas, J. Alloys Compd. 303-304, 198 (2000)

${ }^{5}$ G. Blasse and A. Brill, J. Chem. Phys. 47, 5139 (1967).

${ }^{6}$ X. Zeng et al., J. Cryst. Growth 271, 319 (2004).

${ }^{7}$ E. van der Kolk, P. Dorenbos, J. T. M. de Haas, and C. W. E. van Eijk, Phys. Rev. B 71, 045121 (2005).

${ }^{8}$ L. Bøtter-Jensen, E. Bulur, G. A. T. Duller, and A. S. Murry, Radiat. Meas. 32, 523 (2000).

${ }^{9}$ C. J. Howard, B. J. Kennedy, and B. C. Chakoumakos, J. Phys.: Condens. Matter 12, 349 (2000).

${ }^{10}$ E. van der Kolk and P. Dorenbos (unpublished).

${ }^{11}$ E. van der Kolk, S. A. Basun, G. F. Imbusch, and W. M. Yen, Appl. Phys. Lett. 83, 1740 (2003).

${ }^{12}$ E. van der Kolk, P. Dorenbos, C. W. E. van Eijk, S. A. Basun, G. F. Imbusch, and W. M. Yen, Phys. Rev. B 71, 165120 (2005).

${ }^{13}$ C. Pedrini, D. S. McClure, and C. H. Anderson, J. Chem. Phys. 70, 4959 (1979).

${ }^{14}$ D. S. McClure and C. Pedrini, Phys. Rev. B 32, 8465 (1985).

${ }^{15}$ C. Pedrini and F. Rogemond, J. Appl. Phys. 59, 1196 (1986).

${ }^{16}$ P. Dorenbos, A. J. J. Bos, and C. W. E. van Eijk, J. Phys.: Condens. Matter 14, 199 (2002).

${ }^{17}$ J. Fleniken, J. Wang, J. Grimm, M. J. Weber, and U. Happek, J. Lumin. 94, 465 (2001). 